\title{
Case Report \\ Idiopathic Superior Mesenteric Vein Thrombosis Resulting in Small Bowel Ischemia in a Pregnant Woman
}

\author{
Hao Lin, ${ }^{1}$ Chih-Che Lin, ${ }^{2}$ and Wan-Ting Huang ${ }^{3}$ \\ ${ }^{1}$ Department of Obstetrics and Gynecology, Kaohsiung Chang Gung Memorial Hospital and Chang Gung University \\ College of Medicine, Kaohsiung City 833, Taiwan \\ ${ }^{2}$ Department of Surgery, Kaohsiung Chang Gung Memorial Hospital and Chang Gung University College of Medicine, \\ Kaohsiung City 833, Taiwan \\ ${ }^{3}$ Department of Pathology, Kaohsiung Chang Gung Memorial Hospital and Chang Gung University College of Medicine, \\ Kaohsiung City 833, Taiwan
}

Correspondence should be addressed to Hao Lin, haolin@adm.cgmh.org.tw

Received 24 June 2011; Accepted 17 July 2011

Academic Editors: B. Coroleu and C. Giorlandino

Copyright (C) 2011 Hao Lin et al. This is an open access article distributed under the Creative Commons Attribution License, which permits unrestricted use, distribution, and reproduction in any medium, provided the original work is properly cited.

\begin{abstract}
Background. Small bowel ischemia due to superior mesenteric vein thrombosis (MVT) is rare during pregnancy. However, additional precipitating factors should usually be identified. Case. A 31-year-old woman, pregnant at 34 weeks, was sent to the emergency department because of acute peritonitis. An emergency exploration revealed a segmental gangrene of the small intestine without any mechanical obstruction. Together with the termination of pregnancy, resection of the damaged small bowel was performed, and an end-to-end enterostomy was followed. Based on the operative and pathological findings, small bowel ischemia might be attributed to superior mesenteric vein thrombosis. Conclusion. Hypercoagulation state normally found in pregnant women is believed to lead to this catastrophic condition without other precipitating factors.
\end{abstract}

\section{Introduction}

Small bowel ischemia is a relatively uncommon disorder; the primary causes are diverse and can be grouped into five major categories: (1) strangulation, (2) low-flow states (arrhythmia, sepsis, shock), (3) embolus or thrombosis of superior mesenteric artery, (4) superior mesenteric vein thrombosis (MVT), and (5) miscellaneous. Due to vague clinical presentation and the lack of specific diagnostic tests, early diagnosis for intestinal ischemia is difficult, resulting in significant morbidity and mortality. We herein report a case of a 31-year-old woman who was presented with the signs and symptoms of peritonitis at 34-week gestation. Surgical exploration revealed gangrene in the small intestine without mechanical obstruction. Hypercoagulable state is normally found in pregnant women, which was believed to result in superior MVT and then intestinal ischemia. To the best of our knowledge, this is the second report to describe such a rare disease developing during pregnancy, without evidence of other precipitating factors.

\section{Case Report}

At 34 weeks of gestation, a 31-year-old gravida 3 para 1 woman was referred to our emergency department from a local clinic owing to progressive epigastric pain associated with nausea and vomiting for 3 days. She had no significant medical or surgical history and no history suggestive of thromboembolism. She had never used any oral contraceptives or other hormonal preparations. Upon arrival, her vital signs were stable without fever (BP $120 / 78 \mathrm{mmHg}$, pulse rate $88 / \mathrm{min}$, respiratory rate $19 / \mathrm{min}$, and body temperature $\left.37.1^{\circ} \mathrm{C}\right)$. The hematologic examination revealed marked leukocytosis (WBC 24,200/CMM) and relative intravascular depletion (hematocrit 44.5\%). Coagulation profile and biological tests were within normal limits. Obstetric ultrasound showed a normal male fetus compatible with his gestational age. In addition, a mild fatty change in the liver and a moderate amount of ascites were also noted. The fetal monitor showed that the uterus contracts every 10 minutes. She was initially kept for conservative treatment 


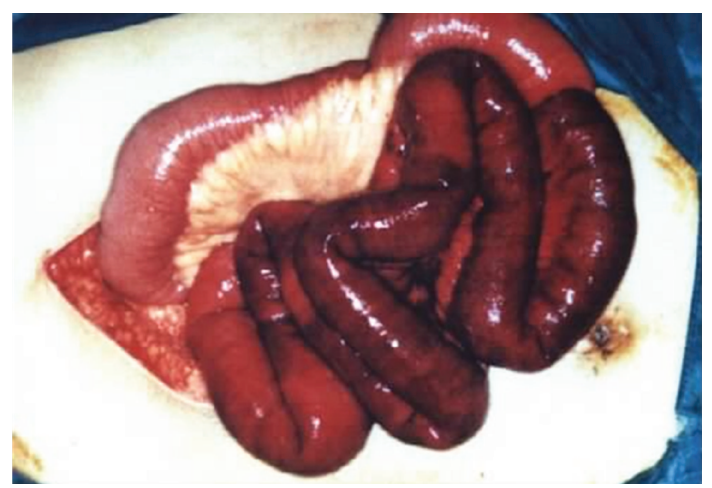

Figure 1: Segmental gangrene of the small intestine without any obstruction and perforation.

(fasting, nasogastric suction) and absolute bed rest under the suspicion of acute gastroenteritis and preterm labor. However, signs of acute peritonitis gradually developed within 6 hours after admission. Thus, an emergency exploration was performed to find a segmental gangrene of the small intestine with 1,500 mL of serosanguineous peritoneal fluid, but no frank obstruction and perforation was identified (Figure 1). The premature baby, at 1,850 grams, was then delivered via cesarean section, with an Apgar score of 4 at one minute and 6 at five minutes. The gangrenous small intestine was resected, and a primary anastomosis was performed. Her postoperative course was uncomplicated, and she was discharged one week after surgery. The outcome of the baby was also excellent without any sequela after intensive care. The pathologic examination revealed mucosal denudation, submucosal edema and hemorrhage, and inflammatory infiltration in the muscularis propria. The intramural and mesenteric vessels in the specimen were patent with blood stasis (Figure 2). Screens for inherited thrombotic disorders (protein S, protein C, antithrombin III deficiency, and factor $\mathrm{V}$ Leiden mutation) for the patient were done after surgery to demonstrate negative results. Four years later, the patient was pregnant again and delivered at term without any thromboembolic event throughout the course. Till now she has been recurrence-free for 7 years without anticoagulant therapy.

\section{Discussion}

Small bowel ischemia occurs very infrequently during pregnancy or puerperium. After searching the MEDLINE, only 16 cases of small bowel ischemia are reported to demonstrate the association of its occurrence with pregnancy to date. Among them, 5 cases $(31.2 \%)$ were caused by the volvulus of the bowel [1-5], one $(6.3 \%)$ by diverticulitis [6], one $(6.3 \%)$ was attributed to cocaine abuse [7], and the remaining 9 cases $(56.2 \%)$ were induced by superior MVT [8-16]. Generally an uncommon type of small bowel ischemia, superior MVT, precipitates more than half of the cases in pregnant women. Precipitating factors associated with MVT may include portal hypertension, postoperative state, trauma, neoplasm, inflammatory bowel diseases,

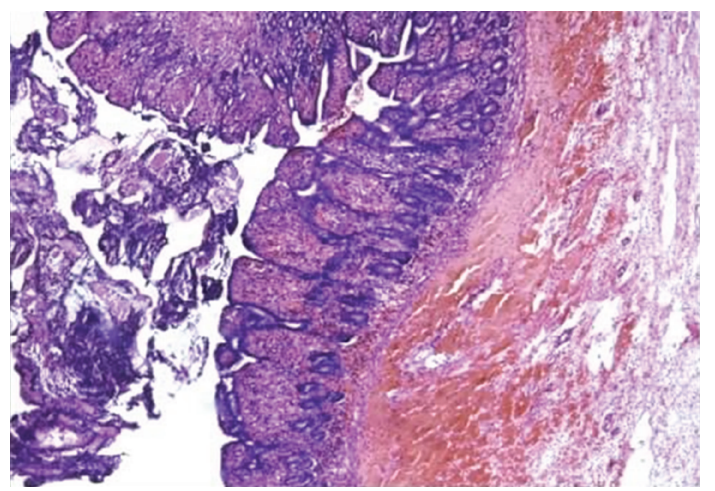

FIGURE 2: Presence of well-formed surface exudates of neutrophils and fibrin (left) with mucosal and submucosal hemorrhage (right) to reveal the ischemic change of small bowel. (H\&E stain, 100x).

use of estrogen-containing compounds, polycythemia vera, hemoglobinopathies, and hypercoagulable states resulting from protein S, protein C, and antithrombin III deficiencies [17]. Through the literature review, additional precipitating factors could be identified in 8 of the 9 pregnant women with small bowel ischemia due to superior MVT: 2 had mistakenly used oral pills during pregnancy $[8,9], 2$ had known history of hypercoagulopathies [10,11], 1 developed MVT soon after elective cesarean section [12], 1 had CMV infection complicated further by toxic megacolon [13], 1 had history of chronic idiopathic MVT [14], and 1 had hemoglobinopathy [15]. Only one case in the literature and our case possess no precipitating factor to develop MVT [16].

Early diagnosis of superior MVT is very difficult due to the nonspecific signs, symptoms, and laboratory results. Almost all patients present with abdominal pain, nausea and vomiting, and leukocytosis with elevated hematocrit, but these are not helpful for diagnosis [17]. Today, the diagnosis of superior MVT is established by a high index of clinical suspicion and noninvasive imaging of the abdomen by ultrasonography, computed tomography, or magnetic resonance imaging (MRI) [18]. The correct diagnosis can be confirmed at laparotomy. Most patients undergo segmental resection of the bowel because of late diagnosis and immediate anticoagulant therapy. The microscopic features of superior MVT include hyperemia, hemorrhage, and edema of the intestinal wall. Mucosal destruction alone occurs in early and less severe lesions, and transmural infarction occurs in the most severe cases. Fresh and organized thrombi extrude from the veins, while arteries are constricted but patent [19]. Although our patient had a similar clinical presentation and laboratory findings, superior MVT was not included in our initial differential diagnosis. Therefore, we did not focus on the mesenteric venous flow or thrombus while performing ultrasonography. Diagnosis was made at laparotomy with the appearance of typical segmental gangrene of the small intestine in association with serosanguineous peritoneal fluid [18]. Microscopic findings of the specimen were typical, except that no thrombi were identified. Whether or not the "presence of thrombi" is essential for the diagnosis of superior MVT remains controversial and requires further investigation. 
Once the diagnosis of superior MVT is made, the immediate use of anticoagulation is indicated due to the high recurrence rate. In patients with inherited hypercoagulable disorders (i.e., protein S, protein C, antithrombin III deficiencies, and factor V Leiden mutation), life-long anticoagulation is warranted. For patients with reversible predisposing causes, at least 6 months of anticoagulation is recommended [18]. Though our patient has been recurrence-free for 7 years after surgery without anticoagulation, the need of prophylactic anticoagulant in this idiopathic etiology still needs further investigation.

In conclusion, it is suggested that in a pregnant woman with a clinical presentation of acute abdomen, even with the absence of the other known risk factors, superior MVT should be included in the differential diagnosis because the pregnancy itself should be considered as an important background etiological factor.

\section{Conflict of Interests}

No conflict of interests are reported by any author.

\section{References}

[1] M. Sivasuriya and S. Y. D. C. Wickramasinghe, "Volvulus of the small bowel complicating mid-trimester pregnancy," Australian and New Zealand Journal of Obstetrics and Gynaecology, vol. 19, no. 4, pp. 247-248, 1979.

[2] J. R. Wax and T. L. Christie, "Complete small-bowel volvulus complicating the second trimester," Obstetrics and Gynecology, vol. 82, no. 4, pp. 689-691, 1993.

[3] L. H. Wanetick, F. P. Roschen, and J. M. Dunn, "Volvulus of the small bowel complicating pregnancy," Journal of Reproductive Medicine, vol. 14, no. 2, pp. 82-83, 1975.

[4] M. Stavorovsky, A. Iellin, M. David, and S. Weintraub, "Midgut volvulus with secondary thrombosis of superior mesenteric vessels in a pregnant woman," Surgery, vol. 81, no. 3, pp. 353-356, 1977.

[5] L. H. Shui, J. Rafi, A. Corder, and D. Mowbray, "Midgut volvulus and mesenteric vessel thrombosis in pregnancy: case report and literature review," Archives of Gynecology and Obstetrics, vol. 283, supplement 1, pp. S39-S43, 2011.

[6] A. B. Alaily, "Gangrene of Meckel's diverticulum in pregnancy due to iron tablet," British Medical Journal, vol. 1, no. 5898, p. 103, 1974.

[7] D. Jawahar, P. J. Leo, N. Anandarao, and B. R. Pachter, "Cocaine-associated intestinal gangrene in a pregnant woman," American Journal of Emergency Medicine, vol. 15, no. 5, pp. 510-512, 1997.

[8] M. Friedman, E. Z. Zimmer, and Z. G. Graubard, "Mesenteric vein thrombosis associated with oral contraceptive administration during pregnancy," Annales Chirurgiae et Gynaecologiae, vol. 73, no. 5, pp. 296-298, 1984.

[9] Z. G. Graubard and M. Friedman, "Mesenteric venous thrombosis associated with pregnancy and oral contraception. A case report," South African Medical Journal, vol. 71, no. 7, p. 453, 1987.

[10] R. Atakan Al, B. Borekci, G. Ozturk, M. N. Akcay, and S. Kadanali, "Acute mesenteric venous thrombosis due to protein $S$ deficiency in a pregnant woman," Journal of Obstetrics and Gynaecology Research, vol. 35, no. 4, pp. 804-807, 2009.
[11] M. Sönmezer, R. Aytaç, L. C. Demirel, and G. Kurtay, "Mesenteric vein thrombosis in a pregnant patient heterozygous for the factor V (1691 G $\rightarrow$ A) Leiden mutation," European Journal of Obstetrics Gynecology and Reproductive Biology, vol. 114, no. 2, pp. 234-235, 2004.

[12] P. McGurgan, M. Holohan, P. Mckenna, and T. F. Gorey, "Idiopathic mesenteric thrombosis following Caesarean section," Irish Journal of Medical Science, vol. 168, no. 3, pp. 164-166, 1999.

[13] F. Zamani, A. Amiri, M. Mohit et al., "CMV infection in a pregnant woman complicated by toxic megacolon and mesenteric vein thrombosis," Turkish Journal of Gastroenterology, vol. 20, no. 3, pp. 234-235, 2009.

[14] C. M. Chan, W. L. Chen, J. H. Chen, Y. L. Wu, and C. C. Huang, "Pregnancy-induced acute intestinal infarction in a woman with chronic idiopathic mesenteric vein thrombosis under regular anticoagulation treatment," Medical Principles and Practice, vol. 18, no. 5, pp. 422-424, 2009.

[15] R. Terzhumanov, E. Uchikova, V. Paskaleva, N. Milchev, and A. Uchikov, "Mesenteric venous trombosis and pregnancy-a case report and a short review of the problem," Akusherstvo $i$ Ginekologiia, vol. 44, no. 3, pp. 47-49, 2005.

[16] T. C. Engelhardt and M. D. Kerstein, "Pregnancy and mesenteric venous thrombosis," Southern Medical Journal, vol. 82, no. 11, pp. 1441-1443, 1989.

[17] C. M. Divino, I. S. Park, L. P. Angel, S. Ellozy, R. Spiegel, and U. Kim, "A retrospective study of diagnosis and management of mesenteric vein thrombosis," American Journal of Surgery, vol. 181, no. 1, pp. 20-23, 2001.

[18] S. Kumar, M. G. Sarr, and P. S. Kamath, "Mesenteric venous thrombosis," The New England Journal of Medicine, vol. 345, no. 23, pp. 1683-1688, 2001.

[19] H. A. Hassan and J. P. Raufman, "Mesenteric venous thrombosis," Southern Medical Journal, vol. 92, no. 6, pp. 558-562, 1999. 


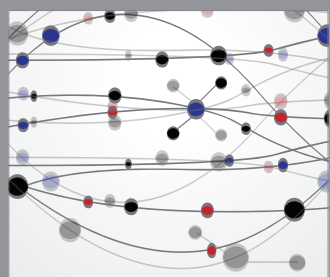

The Scientific World Journal
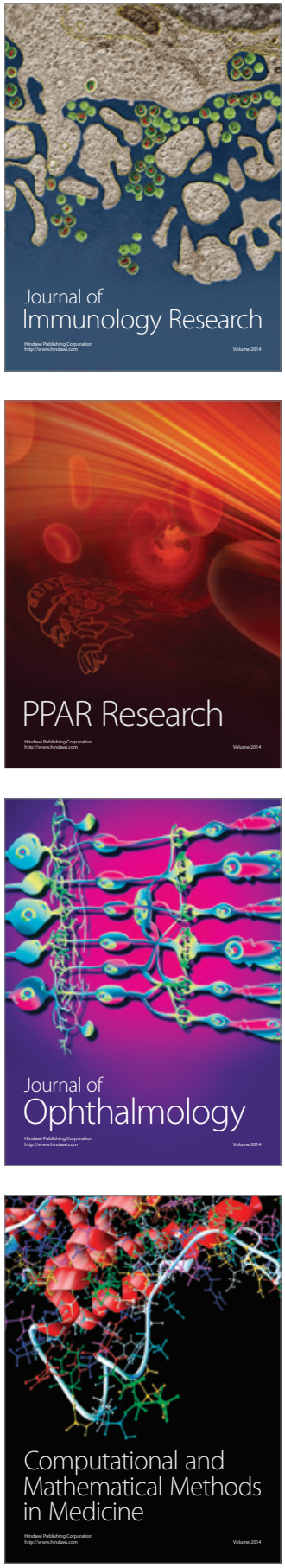

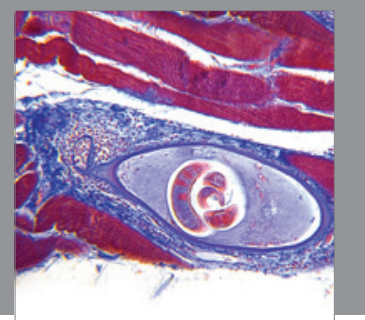

Gastroenterology

Research and Practice
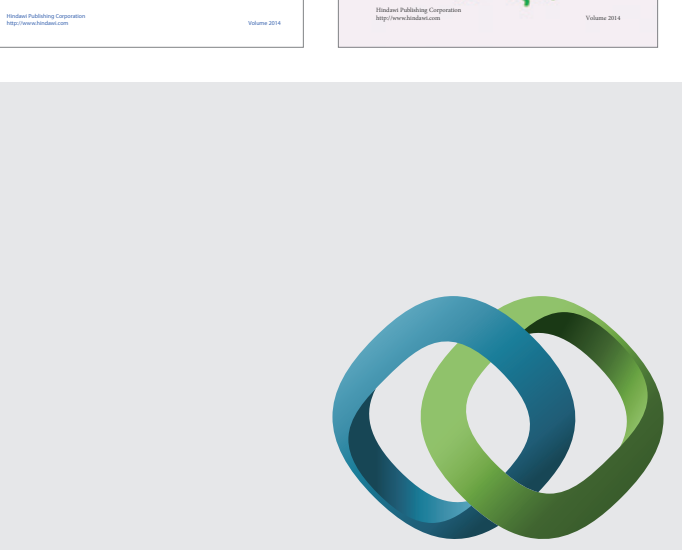

\section{Hindawi}

Submit your manuscripts at

http://www.hindawi.com
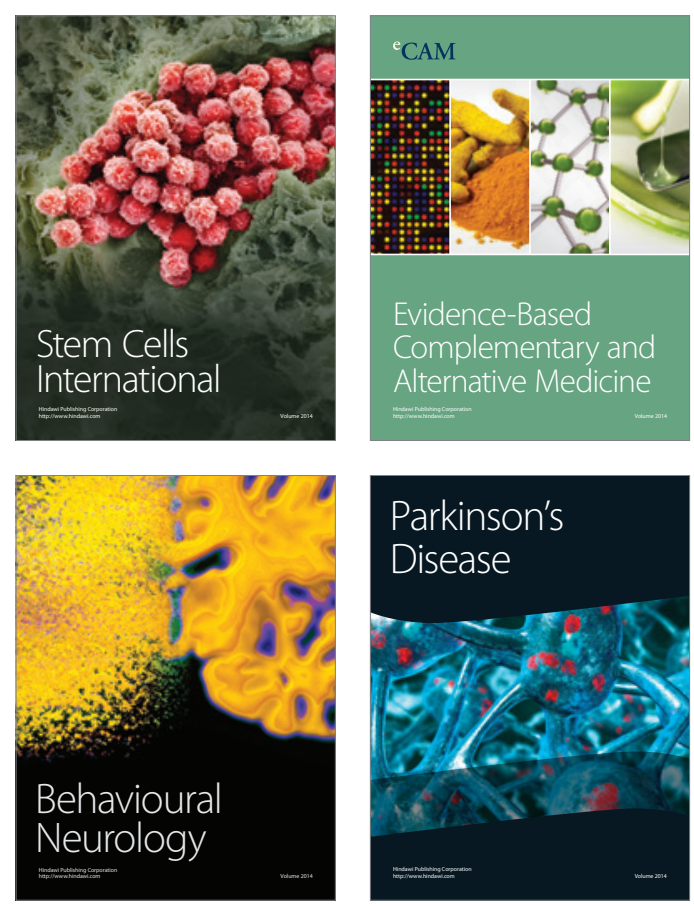

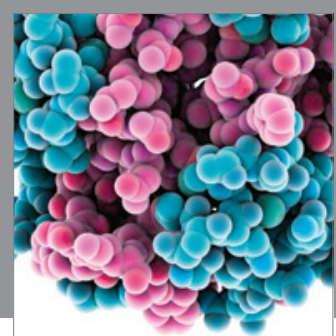

Journal of
Diabetes Research

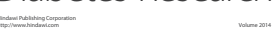

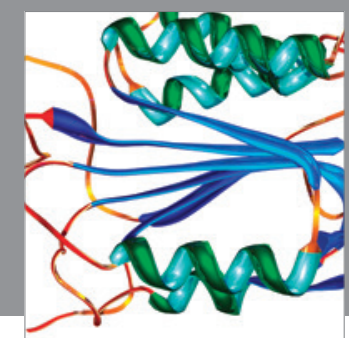

Disease Markers
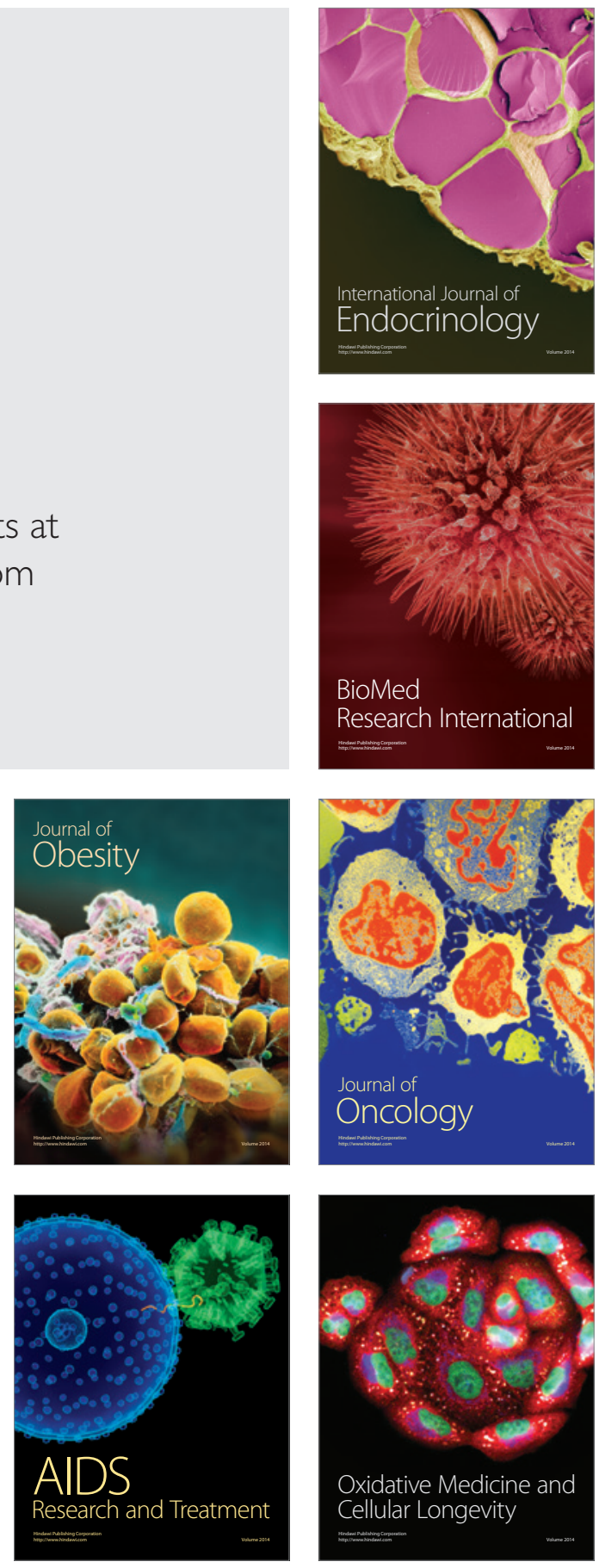